\title{
Order-Disorder Transition in 2D Conserved Spin System with Cooperative Dynamics
}

\begin{abstract}
K. HAŁAGAN* AND P. POLANOWSKI
Department of Molecular Physics, Łódź University of Technology, S. Żeromskiego 116, 90-924 Łódź, Poland

In this work Monte Carlo simulations with usage of dynamic lattice liquid model are presented, instead of the widely used direct exchange or vacancy dynamics, to investigate the dynamics of phase separation phenomenon in spin conserved system with all lattice sites occupied. The dynamic behaviour of domain growth and particle diffusion is discussed for the modified conserved order parameter Ising model. The dynamic lattice liquid model dynamics enables non-locally correlated relaxation dynamics and allows to simulate dense systems in absence of vacancies and parallel treatment of all spins. This approach involves cooperative movement of system elements enabling observation of the order-disorder phase transition in a system with highly correlated motions. Simulations were performed on 2D triangular lattice for several investigated temperatures. Presented results include temporal evolution of domain morphology and diffusion of system elements.
\end{abstract}

DOI: 10.12693/APhysPolA.127.585

PACS: 64.60.De, 05.40.Jc, 64.60.Cn

\section{Introduction}

Order-disorder phase transition in classic Ising system has been intensively investigated for many years [1]. Less attention was put on a case with conserved number of spins (called conserved order parameter model - COP). Conserved spin system is usually referred to alloys or binary mixtures where spinodal decomposition occurs under temperature quench. Rapid quenching below critical temperature of a single phase binary system (composed of two spin species) into a two-phase region causes small (in amplitude) inhomogeneities to evolve into macroscopic domains of two different phases. The system, initially in a homogeneous high-temperature state, becomes inhomogeneous due to order-disorder transition. It is well known from mean-field theory [2] that domain size scales with time as (Lifshitz-Slyozov-Wagner (LSW) growth law [3]):

$$
R(t) \sim t^{x},
$$

with $x=1 / 3$ in the long time limit.

Diffusion of molecules (spins) in the COP models is usually realized in two ways. The first is the Kawasaki dynamics [4], where direct exchange of two randomly selected neighbouring molecules is allowed. The second is vacancy dynamics [5], where exchange is possible only with a neighbouring vacant site. The probability of exchange is commonly assumed to follow the Metropolis rule [6]. The Kawasaki dynamics provides a poor representation of diffusion in a real system because it does not take into consideration the excluded volume condition. The vacancy model gives an unrealistic physical behaviour for high vacancy concentration [7]. Single vacancy model has been proposed [8] to avoid this problem. In this work we propose the use of the dynamic lattice

*corresponding author; e-mail: krzysztof .halagan@p.lodz.pl liquid (DLL) model [9] to simulate the dynamics of orderdisorder transition with highly correlated movements of molecules, reflecting super-cooled spin liquids.

\section{Simulation method}

The dynamics of particles was realized in terms of the DLL model [9], which has been successfully applied for various non-equilibrium physical problems like diffusion limited aggregation [10], evolution of reaction front [11], polymer dynamics [12], gelation process [13] and preliminary studies of phase separation in binary system [14]. In this work a comprehensive explanation of domain growth kinetics and diffusive behaviour in wide temperature range is presented.

In DLL model the molecular system is considered as an assembly of structureless beads representing spins or small molecules oscillating around their current positions with a frequency of attempts to move to the neighboring lattice sites. The location of beads in space is determined by lattice structure. In dense system molecules cannot move separately because all neighboring sites are occupied (excluded volume and local continuity condition). The translation over a distance larger than the vibration range is presented as an attempt to move to a neighboring lattice position. Movement attempts are represented by a randomly chosen unit vectors assigned to each site, pointing to the neighboring site.

An example of a random vector field on a triangular lattice with periodic boundary conditions representing attempts of molecular displacement is shown in Fig. 1. Cooperativeness assumes that attempts to move can be successful only when the local sum of vectors for group of molecules (larger than two) is equal to zero. If not, the attempt fails and molecules remain in presently occupied positions. Collisions (situation (1) in Fig. 1), attempts to create a vacant site (situation (2)), and attempts not participating in a closed loops (situation (3)) are rejected. Only vectors contributing to self-avoiding 
closed paths (loops) enable molecules displacement (situation (4) in Fig. 1). Additionally, movement of every molecule in loops have to be accepted in energetic test with probability given by the standard Metropolis rule [6]. If the move is rejected the whole loop is immobilized. Accepted moves are performed by shifting beads along the closed loops, each bead to a neighboring lattice site. In the long time limit DLL dynamics leads to a Brownian random walk trajectory for every molecule, with jumps distributed randomly in time. One time unit - 1 MCS (Monte Carlo step) consists of few actions: (i) generation of random vector field, (ii) elimination of all attempts not participating in closed loops, (iii) elimination of loops where movement was rejected due to energetic test and (iv) displacement of molecules along remaining loops by one lattice constant.

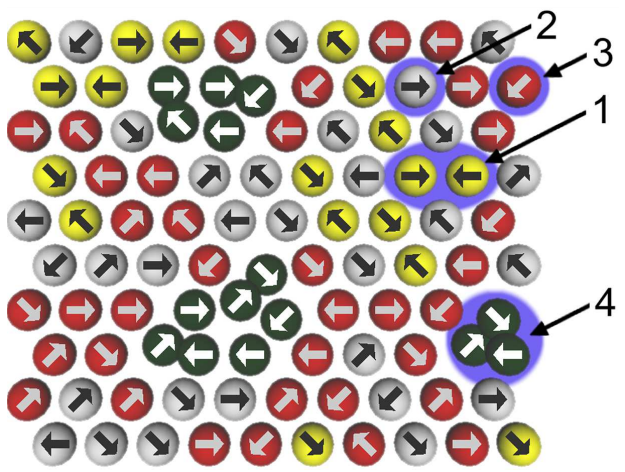

Fig. 1. Schematic representation of a one-phase system in the DLL algorithm. The vector field represents displacement attempts. The elements illustrate various local situations - see the text.

Simulations were performed on $2 \mathrm{D}$ triangular lattice with periodic boundary conditions and $128^{2}$ sites. The results were thermally averaged over several independent runs. At start, an equal number of $\mathrm{A}$ and $\mathrm{B}$ spins were randomly distributed. In the first time step interactions were introduced - the energy of the system was chosen to be in the standard form

$$
E=-\left(J / k_{\mathrm{B}} T\right) \sum_{\langle i j\rangle} \sigma_{i} \sigma_{j}=\left(2 J / k_{\mathrm{B}} T\right) n_{\mathrm{AB}}+\text { const, }
$$

where the sum extends over the nearest neighbors pairs. The symbol $n_{\mathrm{AB}}$ denotes the number of nearest neighbor pairs of $\mathrm{A}-\mathrm{B}$ elements on the lattice, $2 J / k_{\mathrm{B}} T>0$ is the energy of the $\mathrm{A}-\mathrm{B}$ pair ( $T$ is absolute temperature, $k_{\mathrm{B}}$ - the Boltzmann constant), and the symbol $\sigma$ is defined to be +1 for spin $\mathrm{A}$ and -1 for spin $\mathrm{B}$.

\section{Results}

The theoretical value of transition temperature, below which phase ordering occurs, was firstly obtained for triangular lattice by Houtappel [15] as $2 J / k_{\mathrm{B}} T_{\mathrm{c}}=0.5493 \ldots$ Figure 2 presents system configuration for $2 J / k_{\mathrm{B}} T=$ 0.60 (i.e. below $T_{\mathrm{c}}$ ) and increasing number of time steps. A proceeding phase separation can be clearly seen. At the beginning clusters of spins are small with irregular boundaries. After about $10^{3}$ MCS continuous network of domains is formed. Size of formed domains increases nonlinearly with time. A significant number of minority spins can be observed inside majority phase domains. After about $10^{8}$ MCS full system separation is observed. The real computation time at $10^{8}$ MCS was approximately $50 \mathrm{CPUh}$ at $3.7 \mathrm{GHz} \mathrm{CPU}$ frequency for single temperature run. Lower quench temperature would result in smoother phase boundaries and lower concentration of minority spins in majority phase. Presented results are similar to morphologies obtained with other dynamics [8].

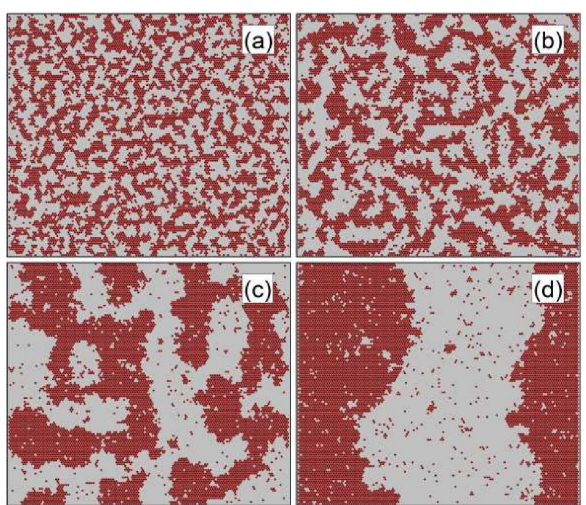

Fig. 2. System configuration for $2 J / k_{\mathrm{B}} T=0.60$ after: (a) $10^{3}$, (b) $10^{4}$, (c) $10^{6}$, and (d) $10^{8}$ MCS.

The analysis of domain morphology and growth kinetics involves determination of the spherically averaged pair correlation function [6]. The first zero of the pair correlation function is taken as a measure of the average domain size $R(t)$. Time evolution of the averaged domain size $R(t)$ for different temperatures is presented in Fig. 3 with evaluated error bars [16]. An early stage of very slow growth can be observed until $t \approx 10^{2}$ MCS. In this stage the morphology of small domains is almost frozen. After that one can clearly see the change of the growth character.

Nevertheless, the theoretical exponent $x=1 / 3$ (Eq. (1)) is not reached even for long times, due to the offset in the growth law caused by the finite system size. For $t>10^{8}$ MCS the growth is stopped because of full separation. Lower temperature result in slower domain growth (with similar exponent). In the case of $2 J / k_{\mathrm{B}} T=0.40$ the growth of clusters is stopped much earlier because temperature is above the critical and full separation is not possible.

Precise analysis of the growth law (Eq. (1)) involves closer examination of the time dependent effective exponent $x_{\text {eff }}$ computed as a logarithmic derivative of $R(t)$ :

$$
x_{\text {eff }}=\mathrm{d}[\log R(t)] / \mathrm{d}[\log t] .
$$

An example of this exponent is shown in inset in Fig. 3 for $2 J / k_{\mathrm{B}} T=0.80$ averaged over 20 independent system configurations. A significant increase of $x_{\mathrm{eff}}$ for $t \approx 10^{2}$ MCS corresponds to the creation of a continuous network of both phases. A drop of $x_{\text {eff }}$ until $10^{3}$ MCS can be explained as stopped growth due to the fact that the size of the domains is small and every displacement of molecules requires reorganization of phase interface. 
After $10^{3}$ MCS the domain size is large enough to enable moves inside domains and the effective exponent starts to increase towards theoretical $1 / 3$.

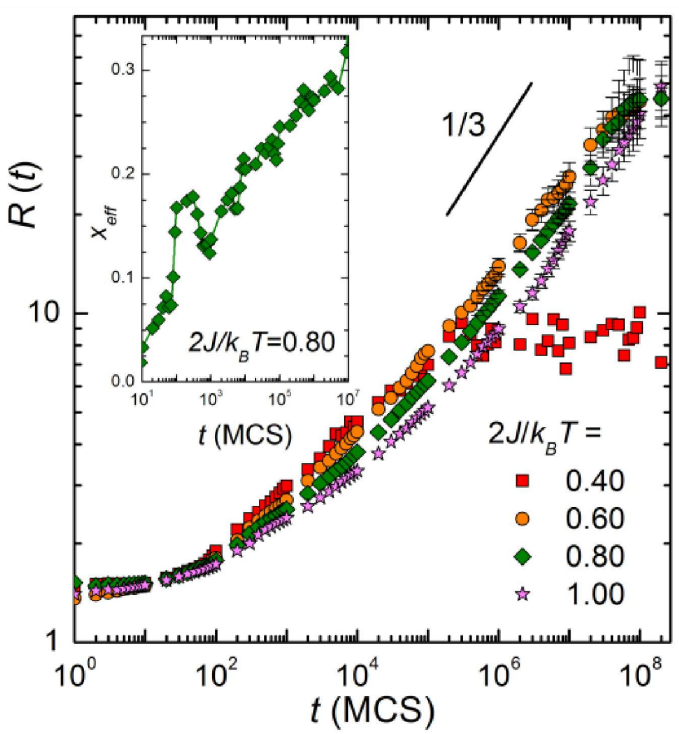

Fig. 3. Average domain size (determined as a first zero of the pair correlation function) measured in lattice units as a function of time for different $2 J / k_{\mathrm{B}} T$. The straight line corresponds to LSW growth exponent. The inset shows effective exponent vs. time.

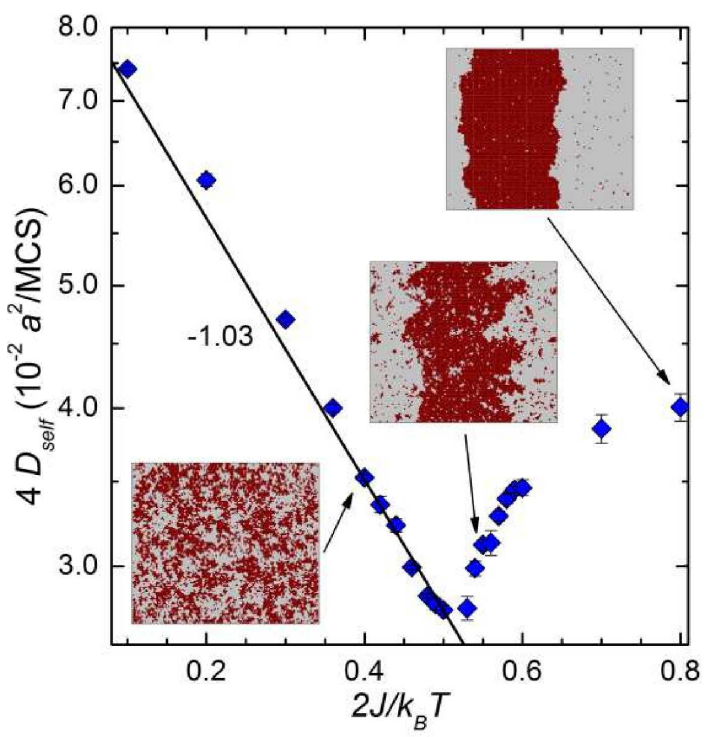

Fig. 4. Arrhenius plot of the logarithm of the selfdiffusion $D_{\text {self }}$ coefficient, in $a^{2} /$ MCS units $(a-$ lattice constant), calculated from mean-squared displacement.

An interesting area in the studies of phase separation kinetics is the analysis of the diffusive behavior of molecules. Self-diffusion coefficients $D_{\text {self }}$ can be obtained using the Einstein equation for equilibrium state

$$
r^{2}(t)=4 D_{\text {self }} t, \quad t \rightarrow \infty,
$$

where $r^{2}(t)$ is the mean-squared displacement. The results are presented in Fig. 4 in a semi-logarithmic scale as a function of $2 J / k_{\mathrm{B}} T$ below and above $T_{\mathrm{c}}$. For the data points above $T_{\mathrm{c}}$ linear fit can be made with a slope $-1.03 \pm 0.01$. This value, closer to 1 than obtained in [14], confirms the well-known Arrhenius-type diffusive behavior with energetic barrier $2 J$. Self-diffusion coefficients increase for temperatures below critical value because in equilibrium state system is fully separated and diffusion coefficients tend back to athermal values. Lower temperature decreases the concentration of minority phase particles inside domains and the movement of majority particles is less disturbed. Further lowering of temperature removes more of the minority atoms from the $\mathrm{A}$ and $\mathrm{B}$ domains but competitive phenomenon limits the increase of $D_{\text {self }}$. The phase interface starts to play a crucial role in this region - an occasional attachment of majority particles to the interface confines their movement.

\section{Conclusions}

The dynamic lattice liquid model is applied to simulate kinetics of order-disorder transition for the modified conserved order parameter Ising system corresponding to super-cooled spin mixtures. Obtained system morphologies are consistent with other studies. The growth exponent $1 / 3$ seems to be reached asymptotically in the long time limit. The time dependent effective exponent reveals local maximum for times between $10^{2}$ and $10^{3}$ MCS corresponding to the formation of the domain network. The behavior of self-diffusion coefficients below the critical temperature is explained by different mechanisms affecting the movement of particles in the equilibrium state.

\section{References}

[1] M. Niss, Arch. Hist. Exact Sci. 63, 243 (2009).

[2] J.W. Cahn, J. Chem. Phys. 42, 93 (1965).

[3] I.M. Lifshitz, V.V. Slyozow, J. Phys. Chem. Solids 19, 35 (1961).

[4] K. Kawasaki, Phys. Rev. 145, 224 (1965).

[5] K. Yaldram, K. Binder, J. Stat. Phys. 62, 161 (1991).

[6] M.E.J. Newman, G.T. Barkema, Monte Carlo Methods in Statistical Physics, Oxford University Press, Oxford 1999.

[7] K. Yaldram, K. Binder, Acta Metall. Mater. 39, 707 (1991).

[8] P. Fratzl, O. Penrose, Phys. Rev. B 50, 3477 (1994).

[9] T. Pakula, J. Mol. Liq. 86, 109 (2000).

[10] P. Polanowski, J. Non-Cryst. Solids 353, 4575 (2007).

[11] P. Polanowski, Z. Koza, Phys. Rev. E 74, 036103 (2006).

[12] P. Polanowski, T. Pakula, J. Chem. Phys. 117, 4022 (2002).

[13] P. Polanowski, J.K. Jeszka, K. Matyjaszewski, Polymer 54, 1979 (2013).

[14] K. Halagan, P. Polanowski, J. Non-Cryst. Solids 355, 1318 (2009).

[15] R.M.F. Houtappel, Physica 16, 425 (1950).

[16] D.A. Huse, Phys. Rev. B 34, 7845 (1986). 